\title{
Histopathologic Review of Calcemic Uremic Arteriolopathy: A Case Series
}

\author{
Jay-V James G. Barit ${ }^{1}$ and Eileen Liesl A. Cubillan² \\ ${ }^{1}$ Section of Dermatology, Department of Medicine, Philippine General Hospital, University of the Philippines Manila \\ ${ }^{2}$ Section of Dermatology, Department of Medicine, College of Medicine and Philippine General Hospital, University of the Philippines Manila
}

\begin{abstract}
Calcemic uremic arteriolopathy or calciphylaxis is an uncommon disorder presenting clinically as skin ischemia and necrosis, and histologically as vascular calcification and thrombosis of dermal and subdermal vasculature. This study described two Filipino females with end-stage renal disease on chronic dialysis with non-healing ulcers on the lower extremities as a result of calcification in the vessels of the dermis and subcutaneous fat with associated fat necrosis. Current understanding of its various histologic features was reviewed for proper diagnosis.
\end{abstract}

Key Words: Calciphylaxis, Vascular Calcification, Calcemic Uremic Arteriolopathy, Chronic Kidney Failure

\section{INTRODUCTION}

Poster presented at the 2018 Research Poster Presentation of the Expanded Hospital Research Office (EHRO), October 8-12, 2018 at the Philippine General Hospital, Manila.

E-poster presented at the $41^{\text {st }}$ Philippine Dermatological Society Annual Convention, November 7-9, 2018 at the EDSA Shangri-La Manila, Philippines.

Corresponding author: Jay-V James G. Barit, MD

Section of Dermatology

Department of Medicine

Philippine General Hospital,

University of the Philippines Manila

Taft Avenue, Manila 1000, Philippines

Telephone: +632 5548400 extension 5105/5106

Email: jayvjamesbarit@gmail.com
Calcemic uremic arteriolopathy (CUA), often referred to as calciphylaxis, is an uncommon, highly morbid disorder presenting with skin ischemia and necrosis. It predominantly occurs but not exclusively, in patients with end-stage renal disease (ESRD). It is characterized histologically by calcification of dermal arterioles, thrombotic vascular occlusion and ischemic skin necrosis. ${ }^{1,2}$ Early data describe a prevalence of $4 \%,{ }^{3}$ but the true prevalence is not known. Current estimates show an incidence rate of 3.5 new cases per 1000 patient years among patients with ESRD on chronic hemodialysis. ${ }^{1}$ Local data is limited to 2 cases of calciphylaxis in Filipino patients with chronic kidney disease, one reported in 2008, ${ }^{4}$ and the second in 2015, as documented in a centralized registry of Philippine dermatologic patients which was started in $2011 . .^{5}$

ESRD is a definite but not a sole risk factor for developing CUA. ${ }^{1}$ It is considered a distinct disorder and not an inevitable complication of chronic kidney disease. ${ }^{6}$ Other reported risk associations include female sex, Caucasian race, diabetes mellitus, obesity, prolonged dialysis vintage, liver disease, various medications (e.g. Vitamin $\mathrm{K}$ antagonists, glucocorticoids, calcium-based binders and Vitamin D analogues), electrolyte derangements from bone-mineral axis disease (e.g. hyperphosphatemia, hypercalcemia and hyperparathyroidism), and various autoimmune (e.g. systemic lupus erythematosus, rheumatoid arthritis) and hypercoagulable conditions (e.g. protein $\mathrm{C}$ and $\mathrm{S}$ deficiency), ${ }^{2,6,7}$ Not all ESRD patients develop CUA, thereby suggesting that it is not just uremia that is required for CUA to occur, but a "second hit" or numerous other events which may be needed to produce overt calciphylaxis. ${ }^{8}$ 
Clinically, CUA presents as reticulate purpura and severely painful plaques and nodules that rapidly progress to nonhealing stellate-shaped ulcers with black eschar. It preferentially involves areas of cutaneous and subcutaneous adiposity, including the abdomen, buttocks, and lower extremities. Histologically, the disorder is characterized by calcification, microthrombosis, and fibrointimal hyperplasia of small arteries and arterioles of the dermis and subcutaneous fat, leading to clinical manifestations of skin ischemia, necrosis, and panniculitis. ${ }^{1,26}$ Current evidence points to active transformation of vascular smooth muscle cells into osteo-chondrocyte phenotypes through upregulation of bonemorphogenic Protein-2 (BMP-2) that actively mineralizes the vascular wall when exposed to elevated serum calcium and phosphate, uremic serum, and parathyroid hormone. ${ }^{9-13}$ Deficiencies and defects in function of inhibitors of vascular calcification, such as Fetuin-A (serum glycoprotein that binds calcium and phosphate $)^{14}$ and matrix glutamic protein (postulated to inhibit BMP-2 and calcium precipitation $)^{10,15}$ are also implicated in its pathogenesis.

Reported outcomes remain poor for CUA patients with mortality rates approaching $30 \%$ and $50 \%$ at 6 and 12 months, respectively. ${ }^{1}$ Sepsis from infected wounds is the leading cause of death in up to $60 \%$ of patients within 1 year of development. ${ }^{1,6}$ Due to the seriousness of the condition, it is important that a clear diagnosis of CUA is established in order to immediately initiate a specific and proper treatment; most commonly used is sodium thiosulfate. ${ }^{2,6}$ The typical histopathologic diagnosis of CUA requires demonstration of calcifications within the media of dermal vessels. ${ }^{6}$ We report two cases of CUA in two Filipino females with ESRD with subdermal vascular and pannicular calcification.

\section{CASE REPORTS}

\section{Case 1}

A 39-year-old Filipino female diagnosed with chronic kidney disease from chronic glomerulonephritis on hemodialysis for 8 years was admitted with a 2 -week history of initially erythematous tender plaques in the right medial thigh which eventually became violaceous with central eschar. Similar lesions appeared on the distal anterior left leg and posterior right leg. The lesions were unresponsive to oral antibiotics. Upon admission, serum chemistry showed normal calcium $(2.23 \mathrm{mmol} / \mathrm{L})$, elevated phosphorus $(2.23 \mathrm{mmol} / \mathrm{L})$ and an elevated calciumphosphate product $\left(75 \mathrm{mg}^{2} / \mathrm{dL}^{2}\right)$. Physical examination showed indurated violaceous plaques with central eschar, with areas of reticulated violaceous patches (Figure 1).A $6 \mathrm{~mm}$ punch biopsy of the center of a violaceous plaque of the right thigh showed extensive dermal necrosis and calcifications of the vessels of the subcutaneous fat with fat necrosis (Figure 2).

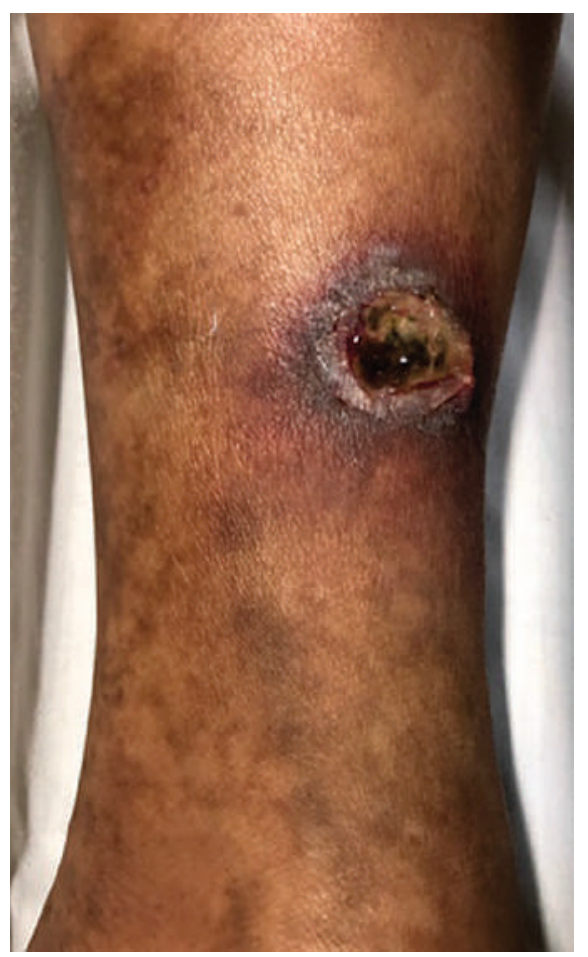

Figure 1. Multiple well-defined irregularly-shaped violaceous plaques with central eschar and surrounding violaceous erythema on the right thigh (A). Multiple reticulated violaceous patches on the left leg, and a well-defined round ulcer with a hemorrhagic base (B). 

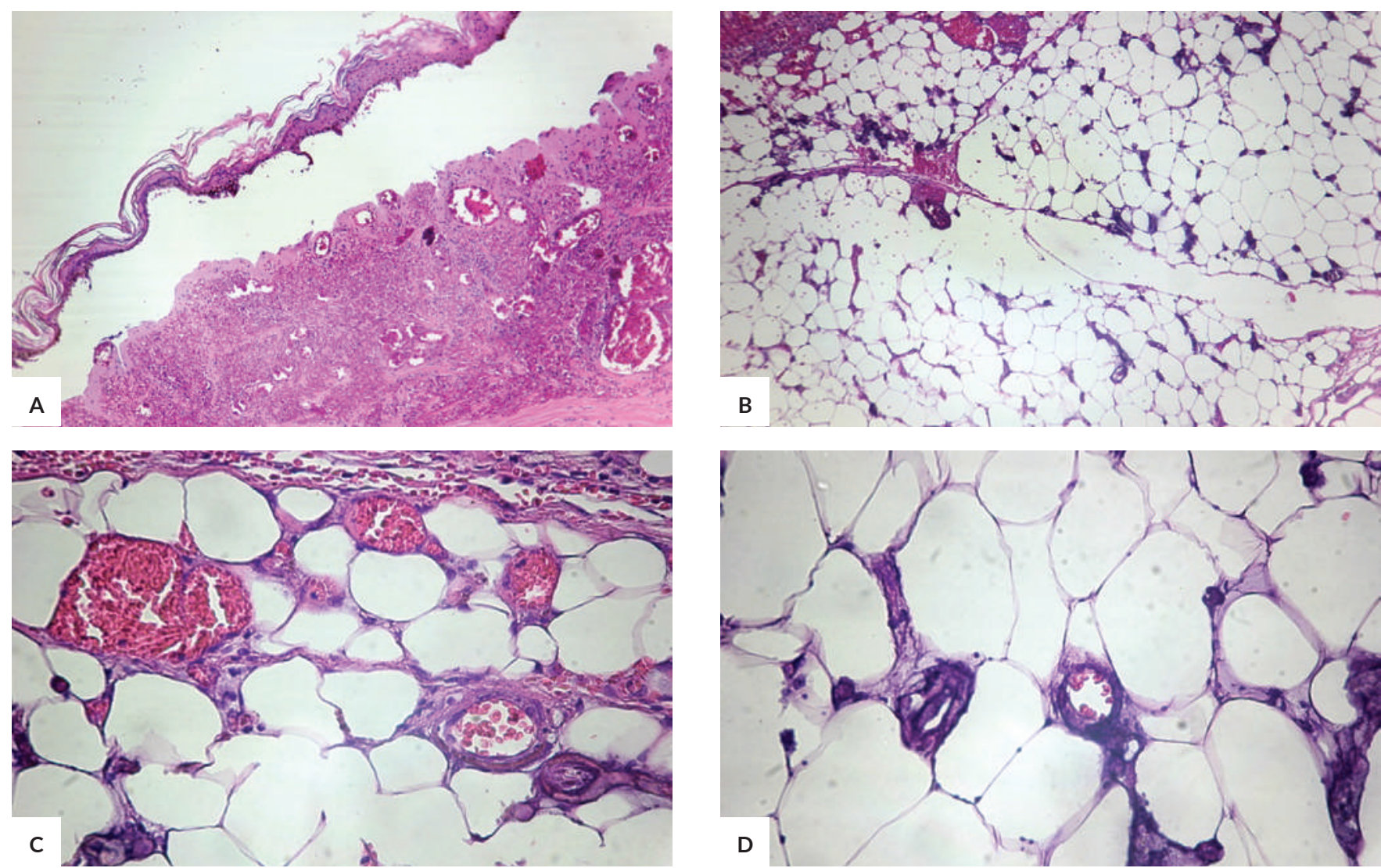

Figure 2. (Hematoxylin-eosin stain). Massive dermal necrosis (A) and calcifications on subcutaneous fat (B) on low power (10x). Higher magnification shows medial calcification of blood vessels in the subcutaneous fat with associated fat necrosis (C\& D).
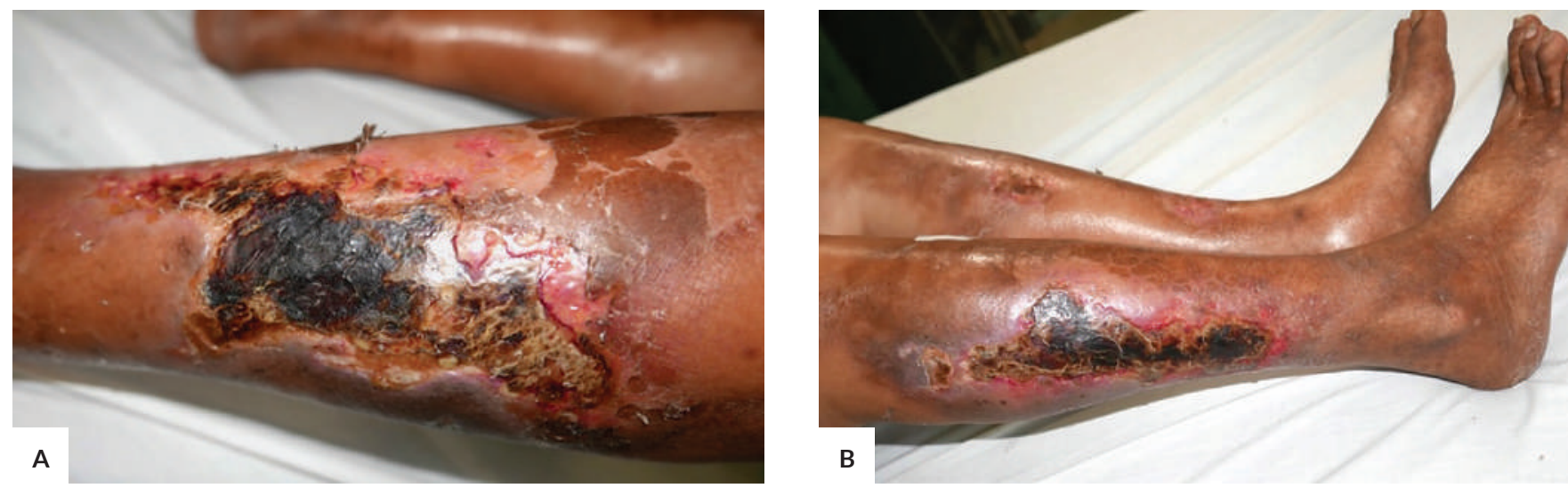

Figure 3. Multiple, well-defined stellate-shaped erythematous ulcer with a dry base with eschar and serous crusts in the left lateral leg (A); no undermining is noticeable. Multiple brown subcutaneous nodules surround the ulcers that were tender to touch (B).

\section{Case 2}

A 40-year-old Filipino female, a known diabetic and hypertensive with chronic kidney disease, on hemodialysis twice a week for 5 years, was admitted for a one-month history of rapidly developing multiple tender, brown to slightly erythematous subcutaneous nodules on both legs that eventually became large and painful non-healing ulcers with necrotic areas. On further dermatologic examination, there were multiple large well defined stellate-shaped ulcers with black eschar at the base, an erythematous, nonundermined border with serosanguinous discharge, and an adjacent well defined irregularly-shaped and erythematous to brown tender subcutaneous nodules (Figure 3 ) on the legs. The rest of the systemic physical examination was unremarkable. 

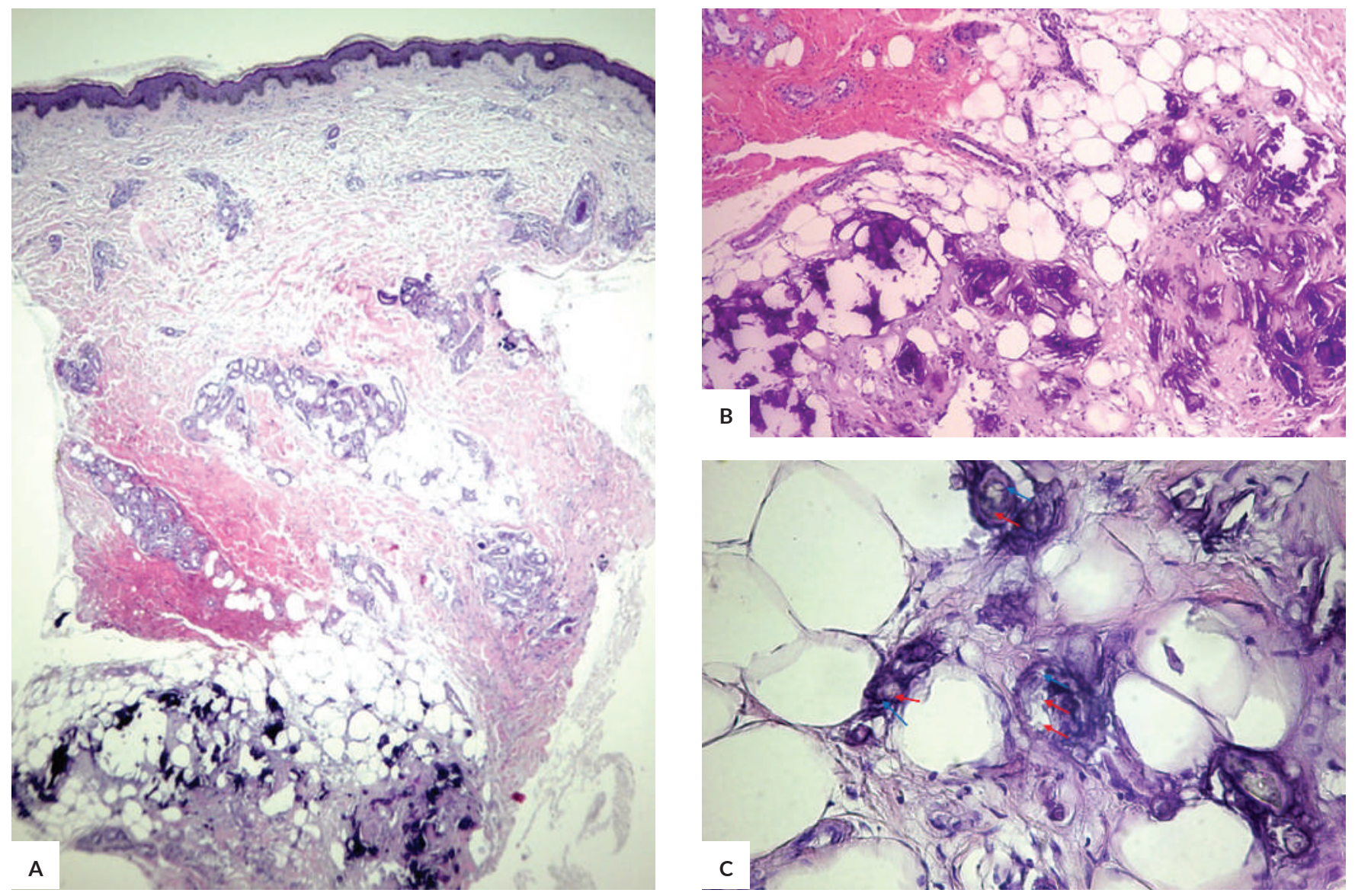

Figure 4. (Hematoxylin-eosin stain) Scanning view (A) showing amorphous basophilic deposits confined to the panniculus (4x). Low power magnification (B) showing subcutaneous fat necrosis, sclerosis and abundant basophilic amorphous material in the panniculus (10x). Red blood cells demonstrated as oval and round refractile material (red arrows) within calcified endothelium (blue arrows), indicating calcified vessels within the panniculus (C, 40x).

Laboratory examinations showed normal serum calcium $(2.25-2.30 \mathrm{mmol} / \mathrm{L})$ and phosphate $(1.33 \mathrm{mmol} / \mathrm{L})$ levels, a non-elevated calcium phosphate product $\left(38 \mathrm{mg}^{2} / \mathrm{dL}^{2}\right)$, and an elevated intact parathyroid hormone $(370 \mathrm{pg} / \mathrm{mL})$ level. A $5 \mathrm{~mm}$ punch biopsy of a subcutaneous nodule near an ulcer margin showed, on routine histology, multiple amorphous basophilic material in the subcutaneous fat. Necrosis of the adipocytes and sclerosis surround the calcifications. Some vessels of the panniculus with refractile red blood cells within the lumen showed subtle medial calcification (Figure 4).

\section{DISCUSSION}

Histologic examination remains the gold standard for diagnosis of $\mathrm{CUA}^{1}$ but is not without inherent risks ulceration, secondary infection, bleeding, propagation of new lesions, and induction of necrosis could occur. ${ }^{2}$ In clinical practice, however, clinicopathologic correlation is recommended to rule out important differential diagnoses such as warfarin skin necrosis, various ulcers (such as stasis, traumatic, or neuropathic ulcers), peripheral arterial disease, vasculitis, and purpura fulminans, among others. ${ }^{1,6} \mathrm{~A} 4$ to 5 $\mathrm{mm}$ punch or a telescoping punch biopsy at the lesion margin is likely to have the best yield and is found to be safer and preferred over incisional biopsy. The center of the ulcer or necrotic areas are considered low-yield sites for biopsy. ${ }^{2}$

CUA histologically appears to be a morphologic spectrum of a calcific thrombogenic microangiopathy, ${ }^{16}$ and no definitive histopathologic criteria exist. Classic descriptions include: a) calcifications of soft tissue and small vessels; b) non-specific intimal proliferation of small vessels which leads to luminal narrowing;c) intravascular fibrin thrombi;d) ischemic necrosis of the skin and subcutis; e) foreign body giant cell reaction to calcium; and f) mixed inflammatory infiltrate of neutrophils and mononuclear cells. In the subcutaneous fat, septal or lobular panniculitis, foci of intralobular calcification and ischemic fat necrosis are generally observed, ${ }^{17,18}$ which was prominent in Case 2.

Based on a three-dimensional analysis of a calciphylactic plaque, the first process to occur in CUA is medial vascular calcification, ${ }^{19}$ specifically of small dermal and subcutaneous 
arterioles. Being a final blood supply point with no alternative collateral blood flow, ${ }^{20}$ this leads to a clinically manifested skin ischemia and eventual necrosis. Patients with ESRD are known to have vascular and metastatic calcification ${ }^{20,21}$ but do not present with CUA. The rapidly progressive and morbid nature of CUA distinguishes it from other forms of vascular calcification, ${ }^{20}$ hence clinical correlation is necessary in all possible cases of CUA.

The prototypic vascular lesion of CUA shows a calcified endothelium with vascular thrombosis and adjacent ischemic damage in the surrounding tissue ${ }^{16}$ such as seen in Case 1. However, classic features in arteriolar calcification have been noted only in $18 \%$ of samples in a retrospective review of 56 patients. ${ }^{22}$ Possible causes behind the low sensitivity include biopsy limitations (sampling error, limited sampling depth, technical processing errors, clinical stage in the process) and similarity of thrombotic vasculopathy in early CUA lesions and hypercoagulable conditions. ${ }^{6}$ Calcific mummification of endothelium may occur but devoid of a thrombus. In the Case 2, calcifications were prominent on the surrounding pannicular tissue (which can be seen in calcinosis cutis) but not readily appreciated within blood vessels. Refractile red blood cells served as the clue to where these calcified vessels were located to fully support the diagnosis of CUA.

Sensitivity can be increased by demonstrating microcalcifications through special stains. ${ }^{6}$ Von Kossa (making calcifications appear black) and Alizarin red (forming an orange-red birefringent product with calcium) stains allow visualization of these subtle microcalcifications in small- to medium-sized arterioles and venules. ${ }^{22,23} \mathrm{~A}$ stippled pattern calcification may be observed using von Kossa staining ${ }^{16}$ for earlier lesions. Von Kossa staining was used to confirm the diagnosis in the local report by Cue and Paliza. ${ }^{4}$ At present, these stains are locally unavailable; but these would have been useful especially for Case 2 wherein the vascular calcification is not as apparent.

The findings for CUA are variable and may be explained by the pathophysiology of the disease and the stage of evolution of the samples in a given time. A retrospective review of cases of CUA categorized findings found in early and late lesions. Both early and late lesions demonstrate vascular calcification, dermal and subcutaneous inflammation, microthrombi formation, and endovascular fibroplastic proliferation. Earlier lesions exhibited dermal collagenous separation, dermoepidermal separation and erythrocyte extravasation, while necrosis and ulceration were seen in later lesions. ${ }^{23}$ Case 1 showed prominent dermal necrosis, suggestive of a late lesion (the necrotic center) while Case 2 had all pathologies confined to the panniculus.

A recent cohort study examining skin biopsies of ESRD patients with and without CUA revealed that CUA histology in ESRD was likely to show diffused thrombi in dermal vessels, focal and diffused calcifications (in middermal vessels and superficial panniculus), and dermal angioplasia. ${ }^{24}$ Perieccrine calcifications in CUA have also been documented ${ }^{22}$, but this cohort study did not find this feature. ${ }^{24}$ In our cases, dermal angioplasia, which the authors have stipulated as a reactive phenomenon to vascular compromise and perieccrine calcifications were not observed. Only the diffused calcifications within vessel walls and extraluminally pointed to CUA in these cases. A small retrospective case control study on hemodialyzed patients purported that calcifications measuring less than $500 \mu \mathrm{m}$ in hypodermal vessels had $86 \%$ sensitivity and $87 \%$ specificity for the diagnosis of CUA, ${ }^{21}$ and this was evident in the two cases presented.

\section{CONCLUSION}

Calcemic uremic arteriolopathy is an uncommon disorder in ESRD patients that is mainly a calcific thrombotic vascular disorder. Histopathologic findings with clinical correlation are important in CUA diagnosis; key findings include calcifications (both distinct and microcalcifications) in dermal and subcutaneous vessels, vascular thrombosis with subsequent ischemic necrosis of the dermis and panniculus in the context of a rapidly evolving morbid disorder. The findings are diverse and represents a morphologic spectrum following its pathophysiology.

\section{Disclaimer}

The views expressed in this article are the authors' own and do not reflect the views of the institution.

\section{Statement of Authorship}

All authors approved the final version submitted.

\section{Author Disclosure}

All authors declared no conflict of interest.

\section{Funding Source}

This paper was funded by the authors. No external funding agency was involved.

\section{REFERENCES}

1. Nigwekar SU. Calciphylaxis. Current Opinion in Nephrology and Hypertension. 2017;26(4):276-81.

2. Nigwekar SU, Kroshinsky D, Nazarian RM, et al. Calciphylaxis: Risk Factors, Diagnosis, and Treatment. Am J Kidney Dis. 2015; 66(1):13346. doi: 10.1053/j.ajkd.2015.01.034.

3. Angelis M, Wong LL, Myers SA, Wong LM. Calciphylaxis in patients on hemodialysis: A prevalence study. Surgery. 1997;122(6):1083-90.

4. Cue PC, Paliza AC. Calciphylaxis: A Report of Two Cases. Journal of the Philippine Dermatological Society. 2008;17(2):80-4.

5. Philippine Dermatological Society Health Information Systems. Philippine Dermatological Society. C 2011 [updated (November, 26, 2017)]; [cited December 6, 2017). Available by request from: pdshis@outlook.com

6. Jeong HS, Dominguez AR. Calciphylaxis: Controversies in Pathogenesis, Diagnosis and Treatment. Am J Med Sci. 2016; 351(2):217-27. doi: 10.1016/j.amjms.2015.11.015. 
7. Nigwekar SU, Zhao S, Wenger J, et al. A Nationally Representative Study of Calcific Uremic Arteriolopathy Risk Factors. J Am Soc Nephrol. 2016; 27(11):3421-3429.

8. Brandenburg VM, Sinha S. Calciphylaxis: Another Piece of the Puzzle. Am J Nephrol. 2017;46(5):427-428. doi: 10.1159/000484545.

9. Weenig RH. Pathogenesis of calciphylaxis: Hans Selye to nuclear factor $\kappa-B$. J Am Acad Dermatol. 2008; 58(3):458-71. doi: 10.1016/j. jaad.2007.12.006 .

10. Ketteler M, Rothe H, Krüger T, Biggar PH, Schlieper G. Mechanisms and treatment of extraosseous calcification in chronic kidney disease. Nat Rev Nephrol. 2011; 7(9):509-16. doi: 10.1038/ nrneph.2011.91

11. Smith ER. Vascular Calcification in Uremia: New-Age Concepts about an Old-Age Problem. Methods Mol Biol. 2016;1397:175-208. doi: 10.1007/978-1-4939-3353-2_13.

12. Wu M, Tang R, Lv L, Ma K, Zhang X, Liu B. Parathyroid hormone induces endothelial-to-mesenchymal transition in human aortic endothelial cells. International Journal of Cardiology. 2011;152.

13. Kramann R, Brandenburg VM, Schurgers LJ, et al. Novel insights into osteogenesis and matrix remodelling associated with calcific uraemic arteriolopathy. Nephrol Dial Transplant. 2013; 28(4):856-68. doi: $10.1093 / \mathrm{ndt} / \mathrm{gfs} 466$.

14. Heiss A, Duchesne A, Denecke B, et al. Structural basis of calcification inhibition by alpha 2-HS glycoprotein/fetuin-A. Formation of colloidal calciprotein particles. J Biol Chem. 2003 Apr 11;278(15):13333-41.

15. Luo G, Ducy P, Mckee MD, et al. Spontaneous calcification of arteries and cartilage in mice lacking matrix GLA protein. Nature. 1997;386(6620):78-81.
16. Magro CM, Simman R, Jackson S. Calciphylaxis: A Review. J Am Col CertifWound Spec. 2011; 2(4):66-72. doi: 10.1016/j.jcws.2011.03.001.

17. Elder DE, Elenitsas R, Murphy BL, Xu X, editors. Lever's histopathology of the skin. 10th ed. Philadelphia: Lippincott Williams \&Wilkins; 2009.

18. Crowson A, Magro CM, Piepkorn MW. Dermatopathology. 3rd ed. Barnhill RL, editor. New York: McGraw-Hill Medical; 2010.

19. Au S, Crawford RI. Three-dimensional analysis of a calciphylaxis plaque: Clues to pathogenesis. J Am Acad Dermatol. 2002; 47(1):53-7.

20. Moe SM, Chen NX. Calciphylaxis and vascular calcification: a continuum of extra-skeletal osteogenesis. Pediatr Nephrol. 2003; 18(10):969-75.

21. Cassius C,Moguelet P, Monfort J, et al. Calciphylaxis in haemodialysed patients: diagnostic value of calcifications in cutaneous biopsy. $\mathrm{Br} \mathrm{J}$ Dermatol. 2018; 178(1):292-293. doi: 10.1111/bjd.15655

22. Mochel MC, Arakaki RY, Wang G, Kroshinsky D, Hoang MP. Cutaneous Calciphylaxis. Am J Dermatopathol. 2013 Jul;35(5):582-6. doi: 10.1097/DAD.0b013e31827c7f5d .

23. Essary LR, Wick MR. Cutaneous calciphylaxis. An underrecognized clinicopathologic entity. . Am J Clin Pathol. 2000; 113(2):280-7.

24. Chen TY, Lehman JS, Gibson LE, Lohse CM, El-Azhary RA. Histopathology of Calciphylaxis: Cohort Study With Clinical Correlations. Am J Dermatopathol. 2017; 39(11):795-802. doi: 10.1097/DAD.0000000000000824. 